\title{
Modelica and Dymola for education in vehicle dynamics at KTH
}

\author{
J. Edrén, M. Jonasson, A. Nilsson, A. Rehnberg, F. Svahn, A. S. Trigell \\ KTH Vehicle Dynamics, SE-100 44 Stockholm, Sweden \\ edren@kth.se,mjonass2@volvocars.com, andnil@kth.se, \\ adamrb@kth.se,fsvahn@kth.se, annika@kth.se
}

\begin{abstract}
Dymola and Modelica have been used in research at KTH Vehicle Dynamics since 2000. With the introduction of new templates and standard components, Modelica has become increasingly accessible for researchers and students in the field of vehicle dynamics and therefore a project was initiated to evaluate its usefulness as an educational tool. The general idea is to introduce Dymola in smaller assignments, aiming to familiarise the students with the basic functions of the tool while demonstrating its use through simulation examples. Four exercises were studied: a truck braking system model, a friction disc clutch model, a controllable torque clutch model and a combined driver and vehicle model. The conclusions from this pilot study were that Dymola offers several ways to enhance the quality of vehicle engineering education, mainly due to the ease of modelling and the rich animation functions which make the simulation exercises more interesting.
\end{abstract}

Keywords: Vehicle Dynamics; Education; Dymola; Modelica.

\section{Introduction}

Dymola and Modelica have been used at KTH Vehicle Dynamics for research work since 2000, see for example [1]. With the Vehicle Dynamics Library [2] (VDL) available, Modelica has become far more accessible for both researchers and students in the field of vehicle dynamics. Because of this, a project was initiated in order to evaluate the current state of Dymola and Modelica as tools for wider use in education at the division. The work presented in this paper was realized as a part of a PhD course, where one of the tasks was to design dedicated exercises to illustrate fundamentals of vehicle dynamics for students.

The strategy for educational introduction of Dymola is to introduce it in different student assign- ments during the final year of education. Four such assignments are described. Generally, the exercises focus on a specific vehicle component or function and uses the features of Dymola to expand the modelling to a wider perspective, thus providing a systems view of vehicle elements while also demonstrating the use of model-based design in vehicle engineering. The specific exercises are described in greater detail in the following sections.

\section{Simulation of an automobile clutch}

In an introductory course in vehicle engineering given at $\mathrm{KTH}$, the students are analysing an automobile friction disc clutch during a vehicle start procedure. The purpose is to study the basic behaviour of the clutch and its effects on the dynamics of the vehicle during vehicle start. The fundamentals of the dynamic system that is analysed are shown in figure 1.

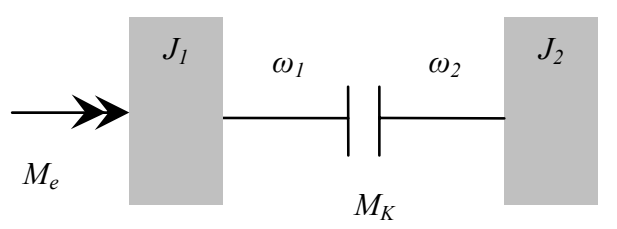

Figure 1: Fundamental driveline model.

Here, $J_{l}$ represents the inertia of the car engine, accelerated by the constant engine torque $M_{e}$. The inertia $J_{2}$ represents the translating mass of the vehicle, transformed here to a rotating inertia. Thus, the rotation of $J_{2}$ corresponds to the motion of the car. The clutch itself is described by the constant torque $M_{K}$, applied between the inertias. External loads such as aerodynamic or rolling resistance are neglected. In the initial position, $J_{l}$ is rotating at a given angular velocity while $J_{2}$ is stationary. At start-up, slipping 
will occur in the clutch until the angular velocity of $J_{2}$ matches that of $J_{l}$.

The equations of motion of this basic setup are straightforward and can be solved analytically. The angular velocities of $J_{1}$ and $J_{2}$ during the start procedure can be seen in figure 2 .

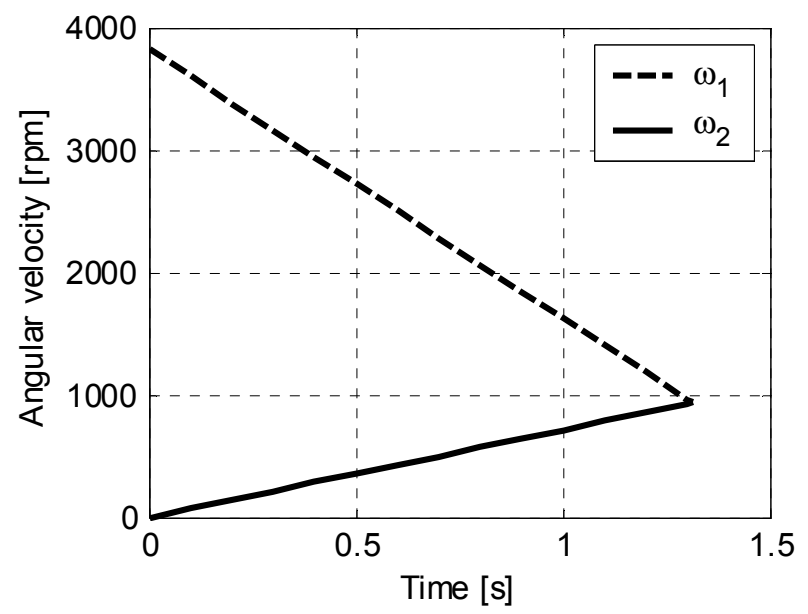

Figure 2: Angular velocities at vehicle start.

The initial, analytical study of the simplified baseline helps to provide a starting point and a basic understanding of the clutch and driveline dynamics. This also establishes good simulation practice as the analytical results serve as a base for comparison with later, more complex simulation models.

After analysing the fundamentals of the clutch and driveline, the students move on to simulating the system using rotational components from the Modelica standard library. This way, a simple but expandable model is built from the ground using physical components. Figure 3 shows the system described in figure 1, as modelled in Dymola.

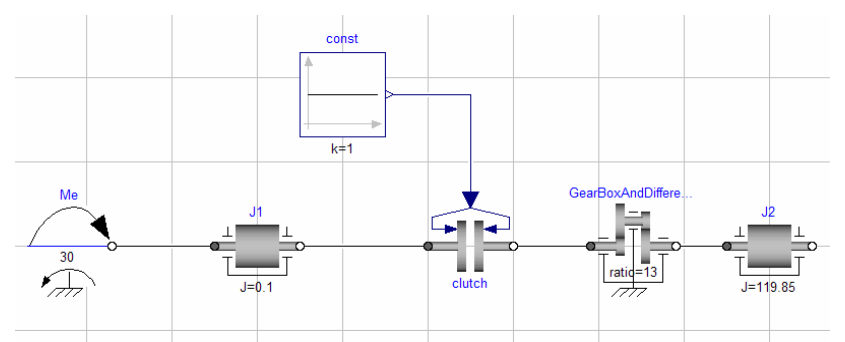

Figure 3: Basic driveline model in Dymola.

With the basic system set up, the analysis can be expanded to cover more complex aspects of the driveline dynamics. Firstly, the clutch model is modified so that the clutch torque is not constant, but ramped from zero to the maximum value in a given time, thus more accurately representing the behaviour of a driver. This makes it possible to study the effects on torque ramping time on the vehicle start, by means of engine rpm and vehicle motion.

Another interesting aspect is the effect of driveline flexibility. Because of the elasticity of the driveshaft, oscillations in the powertrain will occur during vehicle start, leading to a longitudinal vibration in the vehicle known as "shuffle", considered detrimental to ride comfort. Using Modelica, adding a flexible element to the driveline is straightforward and makes it possible to study shuffle vibrations. This expands the initial analysis to include ride comfort aspects as well as vehicle performance.

In the driveline, nonlinearities will also occur in the form of backlashes in the vehicle transmission. Because of backlash, the vehicle will experience a sharp peak in angular acceleration at start up. This is known as "shunt" and is experienced as a longitudinal jerk by vehicle occupants. As the next step in modelling, a backlash element is added to the model, in series with the gearbox and driveline components. With this added functionality, shunt effects in the driveline can be studied and the effect of backlash parameters on shunt magnitude can be studied. The complete model can be seen in figure 4 .

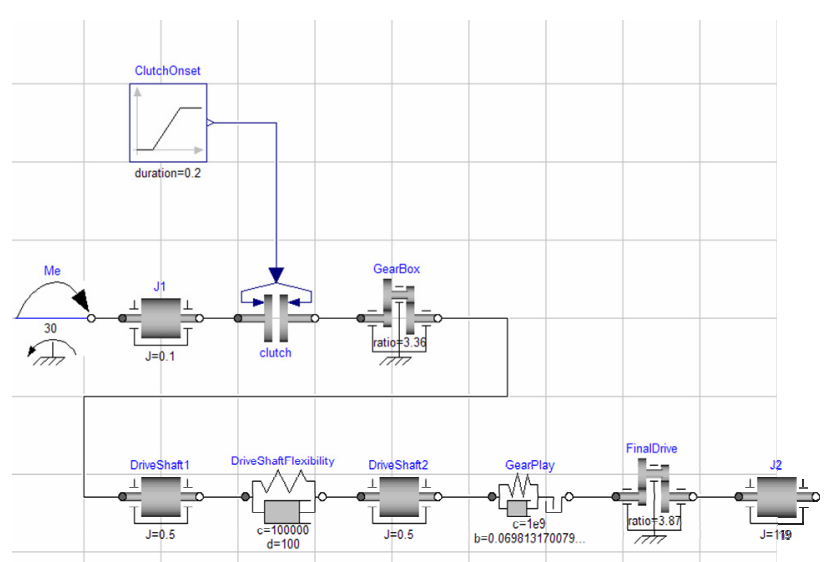

Figure 4: Extended driveline modelling in Dymola.

Figure 5 shows a simulation of clutch engagement with elasticity and backlash included. Compared to figure 2 , the ramped onset of clutch torque is seen in the gradual retardation of $\omega_{1}$ beginning at $0.5 \mathrm{sec}-$ onds. Also, some ripple is visible in $\omega_{2}$ at the same instant, caused by the backlash and flexibility.

The driveline shunt and shuffle can be seen more clearly by further examining the angular acceleration of $J_{2}$. This is shown in figure 6 , where the shunt acceleration and subsequent oscillation can be observed, in this case occurring at the time of clutch lockup. 


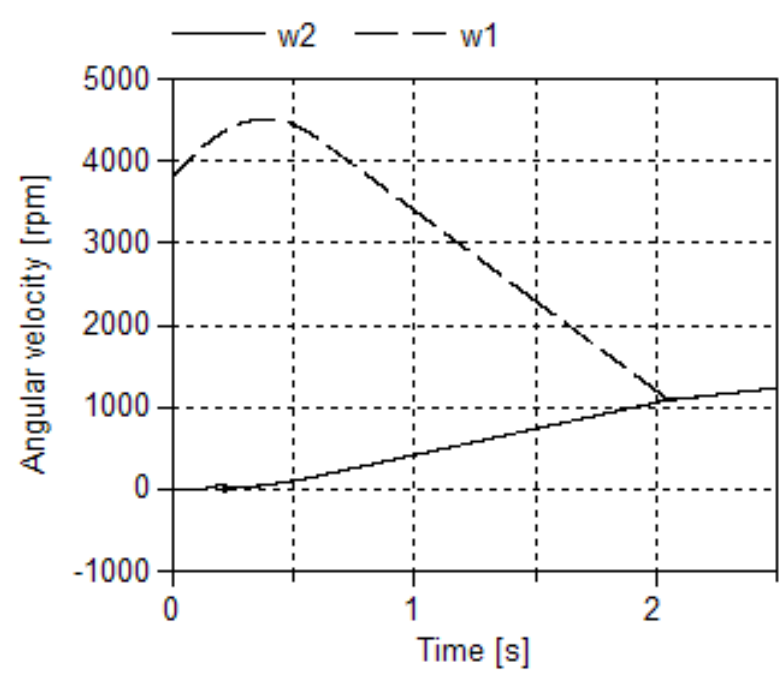

Figure 5: Simulation output from extended model.

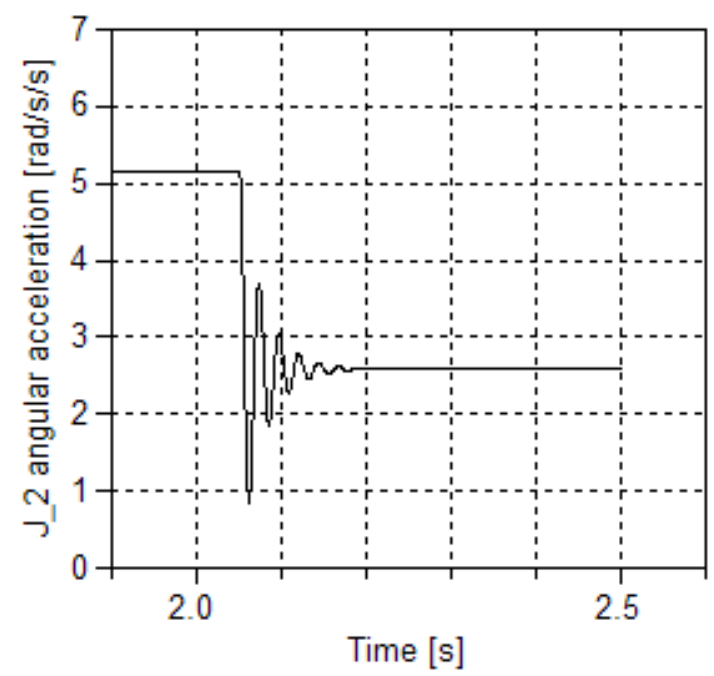

Figure 6: Angular acceleration of $J_{2}$.

This example of a student exercise shows one possibility to use Modelica in education of vehicle engineering. Using the Modelica open library, added complexity can be added to the driveline model without the tedium of lengthy derivations. This makes it possible to simulate a more complete model and thereby provide a deeper insight in the actual driveline dynamics and how it affects vehicle behaviour. However, the equations and algorithms of the model remain accessible because of the open source code and straightforward design of the components. Thus, an understanding of the underlying equations can be maintained although the model itself is made more complex.

\section{Simulation of a truck braking sys- tem}

The task in this assignment, given in the basic ground vehicle engineering course at $\mathrm{KTH}$, is to design the braking system for a truck. It has previously been solved analytically with a tool selected by the student, typically Matlab or Excel. Dymola in addition gives the students a virtual testing environment for verification of their result as well as improved understanding.

The goal of the exercise is to design a braking system for a regular $4 \times 2$ truck to pass legal requirements. To handle both loaded and un-loaded condition, the brake system will need to have a brake pressure regulating valve. Individual vehicle parameters are set for each student. Solving this assignment is of course possible to do with Matlab alone, but together with Dymola the problem is more easily grasped. The idea is to utilize Dymola for virtual testing to visualize for example why it is unwanted to have the rear wheels to lock up.

The whole simulation experiment is based upon the vehicle dynamics library, and is assembled at the highest level. To set up the model all the parameters needed is propagated to be accessible at this level, as seen in figure 7.

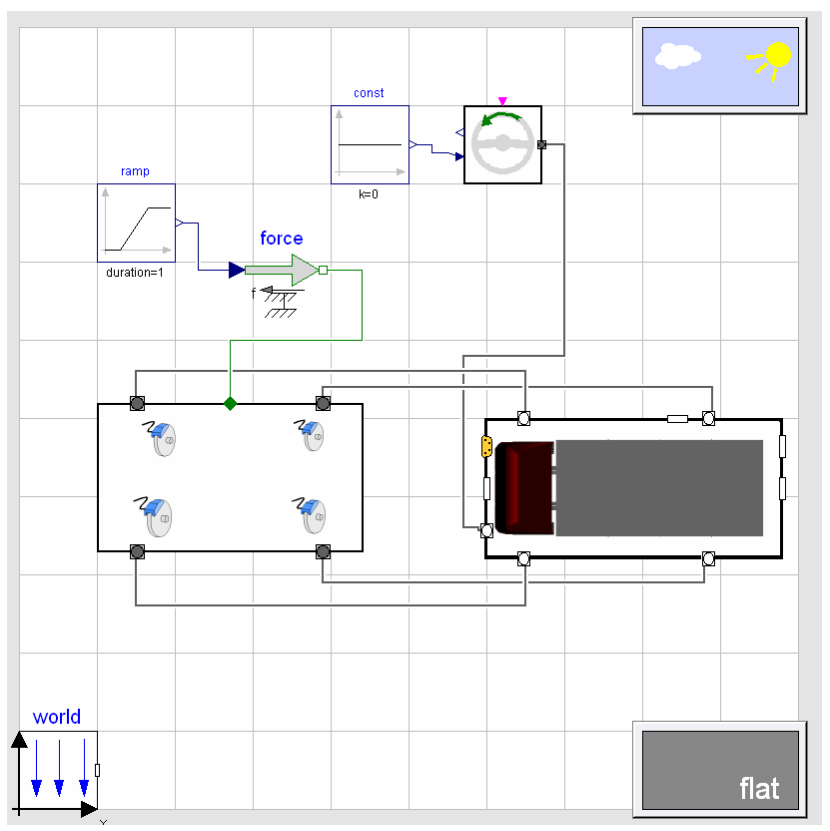

Figure 7: Top level view of the braking experiment.

The Dymola experiment template consists of a truck chassis together with a simple braking system. However using the Dymola model will require some additional calculations, setting up the model to the correct mass and weight distribution. These calculations 
are fairly basic but essential. The Dymola model as a multi body model is very close to the real world as to estimate the total mass and centre of gravity. The idea is to measure axle weights during standstill and at a constant acceleration or deceleration using the multibody model shown in figure 8. From these measurements the total mass and centre of gravity position can be calculated and the model fine tuned. The brake system is put together with exactly the same simple calculations as given in the main task, using gain-blocks. After this point the student will have a good reference to compare against when designing the brake system. One can also see the complexity and difficulties that follow, when beginning the study of more and more complex models that are closer to reality.

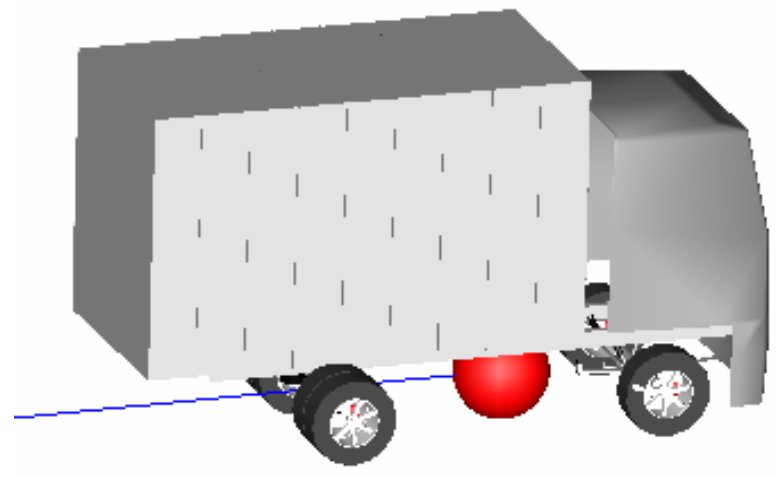

Figure 8: Simulation model used to calculate mass and centre of gravity position.

\section{Torque on demand unit}

This exercise aims to give an insight in advanced modelling and simulation software and its usage as a powerful tool during the development of a vehicle transmission. The vehicle component selected for the exercise, referred to as a torque-on-demand unit (TOD), is a controllable coupling, which is used to distribute propulsion torque between front and rear axle and simultaneously allow for a difference in angular front and rear axle rotational speeds. Classically, TOD systems have gained a lot of attention due to their ability to improve traction, see fore example $[3,4]$. This exercise describes the fundamental functions provided by the TOD and put particular attention to energy consumption and vehicle stability. Emphasise is put on simulation rather than modelling.

The underlying didactic strategy behind this TOD exercise is based on three steps; "Explore", "Execute" and "Evaluate". Necessary qualifications re- quired are basic knowledge in vehicle dynamics and Dymola. Additionally, before the students start the exercise, they need to read a short description of the TOD and how it functions.

\subsection{Explore}

This step aims to provide an understanding of the physics behind the models and students are tempted to open and explore vehicle and subsystem models in Dymola. Emphasis is put on understanding the flow of torque from combustion engine to the hubs. In particular, the students are asked to study the equations for the differentials, which constitute a clear example of that only a few equations are able to describe a relatively complex component. Finally, the students briefly explore the devises modelled for measuring torque, angular speeds and power. This part brings an extra understanding of the physics of the system.

\subsection{Execute}

This step lets the students execute simulations in existing experiments in Dymola. The experiment is based of an urban driving cycle of 18 minutes. The driver gets instructions to drive through the cycle, which means that the driver is forced to follow a desired steering wheel angle and a vehicle velocity. At a particular global position, the vehicle is exposed to split friction while cornering (see figure 9), which threaten vehicle stability. The simulation is repeated with and without the TOD engaged.

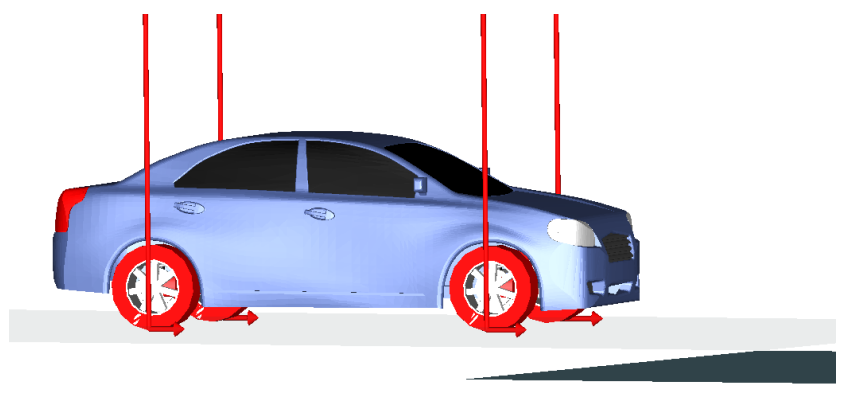

Figure 9: Test drive on split- $\mu$ with a controllable clutch.

\subsection{Evaluate}

The evaluation concerns the energy consumption of the driveline, and in particular, the extra energy consumption caused by friction losses of the four wheel driveline. Power losses are analysed for the individual driveline parts. Finally, an investigation in depth of the split friction event is also made for driveline 
torque distribution and vehicle behaviour. Since one of the most important TOD control goals is to maintain a relatively similar front and rear axle speeds, those will be checked (see figure 10).

\section{Model reduction}

This exercise focuses on analysis by means of linearisation and model-reduction. A complex vehicle model, with and without driver in the loop, is linearised and the results are analyzed using Bode diagrams. Additionally, virtual experiments are performed to find parameter values for simplified models. The nonlinear model is modelled in Dymola, using the Vehicle Dynamics Library (VDL), and the frequency analysis is done in Matlab.

The exercise lets the students learn about how to use the linearisation function in Dymola and also performing virtual experiments to find parameter values of a simple planar single track (bicycle) model. The first part consists of linearising a complex vehicle model from VDL and importing the resulting state space form into Matlab for Bode plot analysis. In the second part the VDL model is simu

lated during specific manoeuvres to determine parameter values for the bicycle model. The bicycle model is then implemented in Matlab and a comparison is made with the complex model through Bode plots. The last part concerns linearisation of a drivervehicle combination in Dymola where some parame- ters of the driver model are varied and the results analyzed in Matlab, again with the use of frequency response diagrams.

The emphasis of this exercise is analysis of vehicle dynamics and not modelling in Dymola. The students are therefore not required to have extensive knowledge of Dymola beforehand. It will, however, give the students an introduction into some advanced usage of the program. To make the exercise as efficient and equal as possible for the students it is suggested that the teachers prepares the required models in a package in advance, and that a tutorial is produced with detailed guidance through the different steps. The students should at this stage not be hampered by difficulties of finding and utilizing the different features of the program. Questions about the vehicle dynamical aspect of the exercise should be interspersed throughout the tutorial, which the students present to the teacher afterwards.

\subsection{Linearisation of vehicle model}

The first task is to use Dymola to linearise a complex vehicle model and import it into Matlab in the form of a state space model. Of interest in characterizing the handling properties of a vehicle is typically to study the response of the lateral acceleration and yaw angular velocity (yaw rate) to inputs from the steering wheel. The model is therefore going to be analyzed through a frequency response diagram.

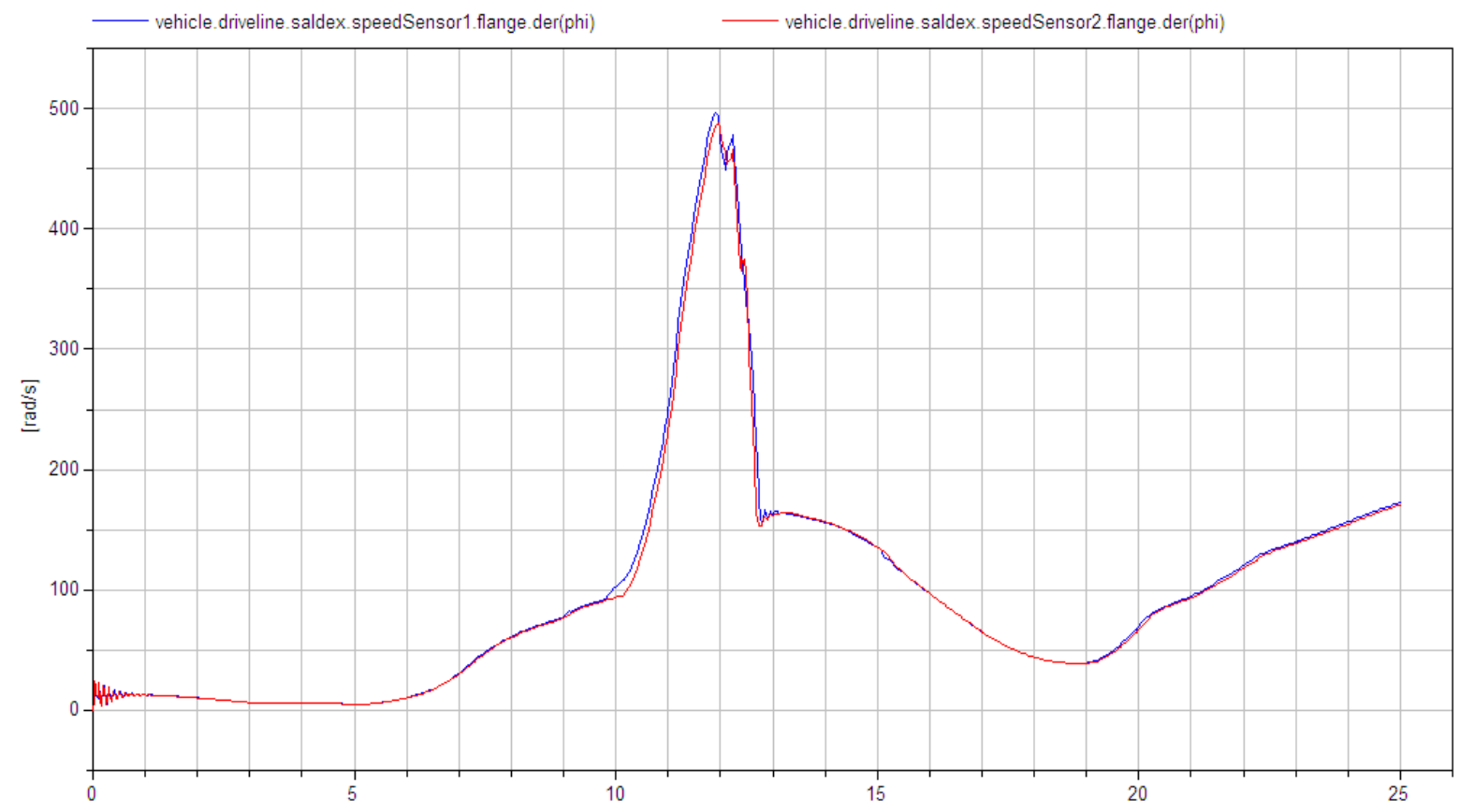

Figure 10: Input and output speeds of the controllable clutch as the vehicle is passing a split- $\mu$ section 
From the VDL the model LEK Pacejka02 is chosen. It represents a small compact car and has 134 states in its dynamical description. The kinematics of the suspension is detailed, including variable toe- and camber angles. The tyre model is a Pacejka Magic Formula model which is suitable for handling analysis.

An input and output has to be defined for the vehicle model to enable frequency response analysis. Based on the OpenLoopDriver model of the VDL, two version of the model are prepared with steering wheel angle as input and lateral acceleration and yaw rate as output respectively. Figure 11 shows one of the models. As can be seen in the figure a constant term is added to the steering angle input to account for an offset in the neutral position of the steering wheel caused by an asymmetry in the steering system.

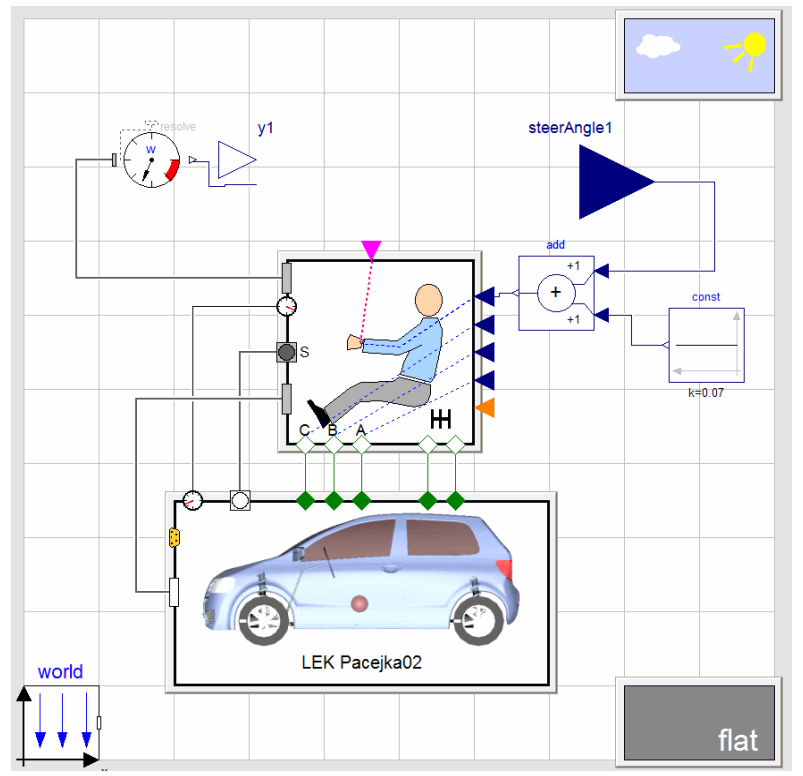

Figure 11: Driver model with defined input/output.

The Linearize command in Dymola uses the initial values of a simulation to linearise about. Therefore it is suggested that a short straight ahead driving simulation is performed where after the command importInitial(); on the command line loads the terminal values of the simulation as initial conditions. Thus linearisation around a steady state condition is assured. The Linearize command is then found from the Simulation menu. In this process, a file named dslin.mat is generated that contains the linearised model. Use tloadlin.m found in the Dymola search path to import the linearised model into Matlab:

$>[\mathrm{A}, \mathrm{B}, \mathrm{C}, \mathrm{D}, \mathrm{xName}, \mathrm{uName}, \mathrm{yName}]=$ tloadlin('dslin');
State space systems of the imported matrixes are made with the ss-function:

$>$ sS_sys $=\operatorname{sis}(A, B, C, D)$;

If the system has several outputs and only one of them is to be used for the state space system, limit C and $\mathrm{D}$ to one of the rows (output parameter index can be identified with yName).

After linearisation and creation of state space systems, Bode plots can be generated and used for analysis, e.g. with the Matlab function:

$>$ bode (ss_sys).

\subsection{Determination of parameters}

The second task is to find parameter values for a two-degree-of-freedom bicycle model which corresponds to the complex model. The governing differential equations are seen in equation 1 ,

$\left(\begin{array}{l}\dot{v}_{y} \\ \ddot{\psi}\end{array}\right)=\left(\begin{array}{cc}-\frac{C_{12}+C_{34}}{m v_{x}} & -v_{x}+\frac{-f C_{12}+b C_{34}}{m v_{x}} \\ \frac{-f C_{12}+b C_{34}}{J_{z} v_{x}} & -\frac{f^{2} C_{12}+b^{2} C_{34}}{J_{z} v_{x}}\end{array}\right)\left(\begin{array}{l}v_{y} \\ \dot{\psi}\end{array}\right)+\left(\begin{array}{l}\frac{C_{12}}{m i_{s}} \\ \frac{f C_{12}}{J_{z} i_{s}}\end{array}\right) \delta_{R}$

(1),

where $v_{y}$ is the later velocity, $\psi$ the yaw angle, $C_{12}$ and $C_{34}$ the cornering stiffness front and back, $m$ the total mass of the vehicle, $v_{x}$ the longitudinal speed, $f$ and $b$ the distance from centre of gravity to front and rear axle respectively, $J_{z}$ the moment of inertia around the vertical axis of the vehicle, $i_{s}$ the steering ratio and $\delta_{R}$ the steering wheel angle.

The value of $L$, i.e. $f+b$, is given to the students. $J_{z}$ of the sprung mass of the vehicle is also given. However, the students are asked to make back-ofthe-envelope calculations of the additional moment of inertia of the unsprung masses. To find the rest of the parameter values a couple of simulations are performed with the vehicle model. First a straight ahead drive is used to find the normal forces on each tyre, from which $f$ and $b$ can be calculated. Then, a steady state cornering simulation is performed with a small steering angle. From the lateral forces and body slip angle the cornering stiffness coefficients can be calculated. The effective steering ratio is measured by making a sinusoidal steering simulation with the ground friction set to a small positive number (zero does not work numerically) and then comparing the steering wheel angle to the wheel angles.

These virtual experiments can principally be done with a real vehicle as well. The measurement equip- 
ment needed is four scales, accelerometers and a device to measure body slip angle. The lateral tyre forces can be calculated from the lateral acceleration.

\subsection{Analysis of linearised models}

Identifying the A, B, C and D matrices of the bicycle model makes the two models ready for a comparative analysis. The frequency response of the two models can be compared for different speeds and the students should note for what frequency range the simple model is a good approximation for the complex model. Figure 12 shows an example of a Bode diagram of the two models with the yaw rate as the output. It can be noted that the bicycle model starts to deviate from the complex model in phase already at $1 \mathrm{rad} / \mathrm{s}$ and in magnitude at about $4 \mathrm{rad} / \mathrm{s}$, but that the shape of the curves are quite accurate up to about $70 \mathrm{rad} / \mathrm{s}$. Students should also note that the simple bicycle model is unable to capture the local peak in phase at about $100 \mathrm{rad} / \mathrm{s}$.

\subsection{Driver model analysis}

The objective of the last exercise is to study effects of changed parameters in a driver model. A linearisation of an existing nonlinear driver-vehicle model is done in order to analyze Bode diagrams of the generated linear model. None of the existing driver model experiments in the current VDL is suitable for analysis without modifications, but the main part of these modifications can be done in advance to shift focus from modelling to analysis. Here a tutorial is suggested to guide the students for the remaining modelling and analysis, since this can improve the understanding of the driver model components with only a small amount of additional effort. A tutorial approach should, compared to an exercise where the student has to solve the modification problem by him or her self, not only be quicker but should also result in identical baseline models for analysis. This gives the students equal opportunities to do analysis with the models.

\subsection{Assembling the driver-vehicle model}

One of the existing experiments in VDL, the ClosedLoopDriver, is used here after it has been modified to enable input of the preview point position and output of (at least) the position of the vehicle. The model is later linearised around zero steering wheel angle, so that the longitudinal distance from the vehicle to the preview point can be assumed to be relatively equal for both global and vehicle coordinates.

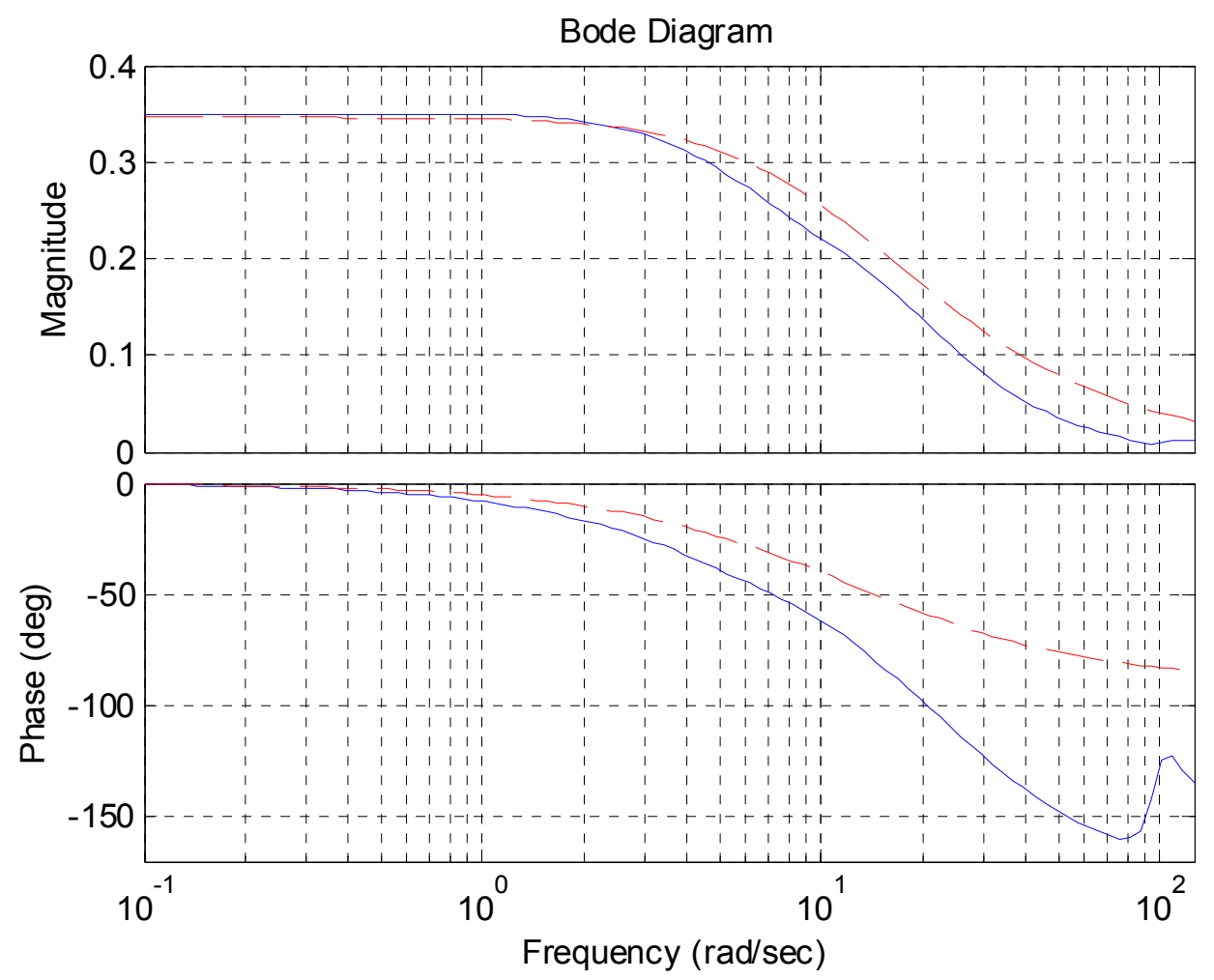

Figure 12: Bode diagram of the complex linear model (solid) and simple bicycle model (dashed). The output is here the yaw rate and input is the steering wheel angle. 
Hence we can use only the lateral component of the preview point and vehicle position as input and output respectively, and thus limit the number of inputs and outputs to two. The use of global reference for both input and output enable analysis of how well the driver-vehicle system will follow the path described by the preview point. To simplify the model, the driver in the ClosedLoopDriver is replaced with a constant speed driver based on the ClosedLoopLateral template. The tracker in the template is modified to allow direct input of lateral position into the steering control calculation since the standard path input taken from spatial time independent coordinates. Direct input of preview point position in vehicle reference frame into the tracker is required for active modification of the preview point position.

At the experiment level, the LEK Pacejka02 vehicle is selected again. In the chassis, the parameter summary resolve point is changed from the centre of the front axis to the vehicle centre of gravity. It is also necessary to specify an approximation of the steering wheel gear ratio to be used by the driver model, and this can be done in the experiment with given data or experimental results.

For the correct input to the tracker block in the driver model, a new text layer block is added with the coordinate transformation from global reference to the vehicle front axis, which requires adding sensors to the top level of the experiment (see figure 13). Possibility to change the preview time is also added as an input to the transformation block. One input has to be added to the experiment, the lateral position of the preview point. Several outputs can be added, i.e. the lateral position of the vehicle that will be used here for analysis of how the drivervehicle system responds to movement of the preview point.

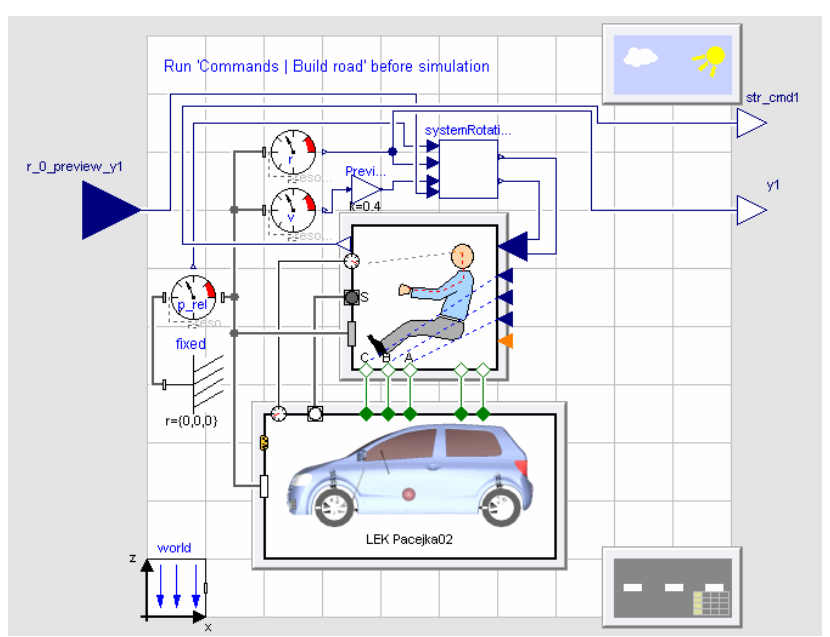

Figure 13: Top level view of driver-vehicle experiment.

\subsection{Analysis of linearised model}

With the model modified it is now possible to export the model for analysis. The system can now be set to different states in the experiments, e.g.:

- In the vehicle, set the velocity to a chosen value to use for the linearisation.

- In the top level of the experiment, change the preview time to analyze the effect of different path tracking strategies.

- Change the steering wheel gear ratio in the driver model to analyze effects of driver interpretation of the vehicle characteristics.

The model can be linearised and exported after the experiment simulation has been executed in the same way as described in Section 2.1. The students should analyze the default setup of the driver model first, e.g. with accurate information about the vehicle at a selected speed, and then try different values to study the effects of the parameters. Bode plots of three different selected preview times are presented in figure 14. The resonance peak is seen to grow for decreasing preview time, which would indicate that the system becomes more nervous. 


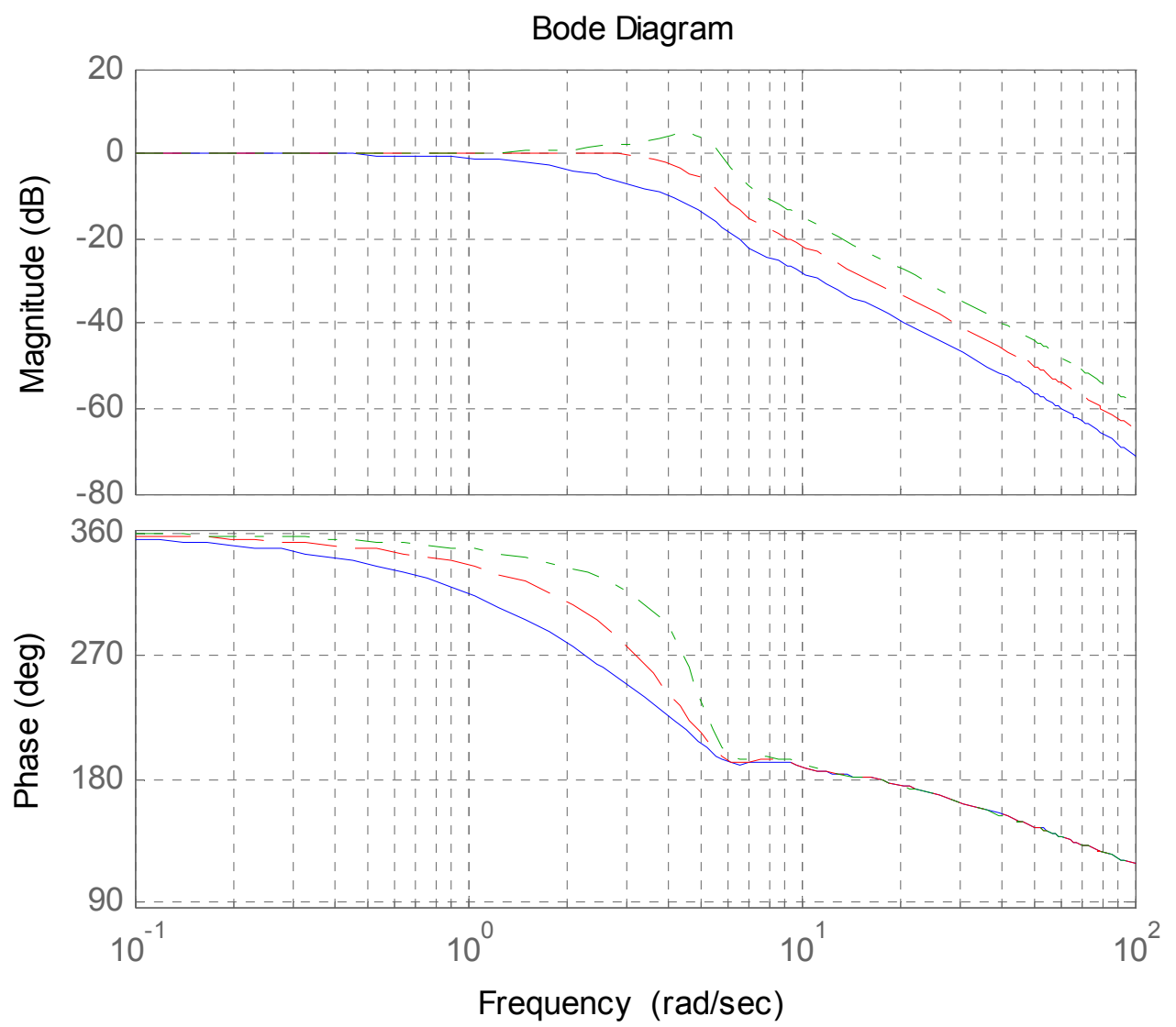

Figure 14: Bode diagram of linearised model, using steering wheel gear ratio 19, speed $30 \mathrm{~m} / \mathrm{s}$, preview time 0.8 (solid), 0.4 (dashed) and $0.2 \mathrm{~s}$ (dash-dotted).

\section{Conclusions}

The evaluation during the course of this project has shown that Dymola and VDL offer many ways to enhance the learning of vehicle engineering. The physical based modelling makes it easy to replace or add components, while the hierarchical structure and icon based model layout give the user a clear overview of the different subsystems and their interconnections. Animations also help to visualise results and clearify physical behaviourThe VDL also contains example models which can be used to perform virtual experiments as a substitute for real life experiments that might be too expensive or time consuming for a student course. Modifying the example models and experiments is also easy, making it straightforward to design student exercises showing various aspect of vehicle dynamics.

The problems with Dymola include the somewhat crude tools for analysis, sometimes making it necessary to export models or simulation results to other tools such as Matlab. Also, the documentation can be found inadequate when confronted with certain problems such as the linearisation process.

\section{References}

[1] Andreasson J., Möller A., Otter M.: Modeling of a Racing Car with Modelicas Multibody Library, in Proceedings of the Modelica Workshop, 2000.

[2] Andreasson, J., Gäfvert M.: The Vehicle Dynamics Library - Overview and Applications, in Proceedings of the 5th international Modelica Conference, 2005.

[3] Mäki R.: Wet Clutch Tribology - Friction Characteristics in Limited Slip Differentials, doctorial thesis, Luleå University of Technology, Sweden, 2005.

[4] Piyabongkarn D., Lew J.Y., Rajamani R., Grogg J.A. and Yuan Q.: On the use of Torque-biasing Systems for Electronic Stability Control, IEEE transaction on control systems technology, Vol. 15, No. 3, pp. 581589, 2007. 